\title{
CD46-associated atypical hemolytic uremic syndrome with uncommon course caused by cblC deficiency
}

\author{
Antonia H. Bouts • Marcus T. R. Roofthooft • \\ Gajja S. Salomons • Jean-Claude Davin
}

Received: 19 June 2010 / Accepted: 1 July 2010 /Published online: 24 July 2010

(C) The Author(s) 2010. This article is published with open access at Springerlink.com

Sirs,

In a recent edition of Pediatric Nephrology, we reported the case of a child with a slowly progressive on-going atypical hemolytic uremic syndrome (aHUS) associated with a mutation in the gene encoding membrane cofactor protein (CD46) [1]. Mutation screening of $C F H, C F I, C 3$ and $C F B$ showed no abnormalities. Serum factor $\mathrm{H}$ and factor I concentrations were $0.55 \mathrm{~g} / 1$ (normal range $0.35-0.59 \mathrm{~g} / \mathrm{l}$ ) and $49 \mathrm{mg} / \mathrm{l}$ (normal range $38-58 \mathrm{mg} / \mathrm{l}$ ), respectively. Mutation screening of CD46 showed a heterozygous missense mutation in exon 11 (c.1058C > T, p.Ala353Val; alternative syntax Ala304Val). The course of this child contrasted with the often observed repetition of acute HUS episodes generally followed by complete recovery of renal function that is presented by most patients with a $C D 46$ mutation [2]. The initial episode was treated with daily plasma exchanges (PE). Although this treatment was initially associated with a complete renal function recovery, over the next 4 years there was a progressive decline in renal function to end-stage renal failure (ESRF) despite

A. H. Bouts $\cdot$ J.-C. Davin $(\bowtie)$

Pediatric Nephrology Department, Emma Children's Hospital,

Academic Medical Center, University of Amsterdam,

Meibergdreef 9,

AZ 1105 Amsterdam, The Netherlands

e-mail: j.c.davin@amc.nl

\section{T. R. Roofthooft}

Beatrix Children's Hospital, Department of Pediatric Cardiology and National Expertise Center for Children with Pulmonary Hypertension, University Medical Center,

Groningen, The Netherlands

G. S. Salomons

Department of Clinical Chemistry, Metabolic Unit,

VU Medical Center,

Amsterdam, The Netherlands prophylactic PEs, with evidence of an on-going thrombotic microangiopathy seen at kidney biopsy. Seventeen months after starting hemodialysis, the patient received a cadaver kidney transplant. Immunosuppression comprised prednisolone, low-dose cyclosporine, mycophenolate mofetil and basiliximab. Plasma therapy was undertaken immediately prior to transplantation, was continued daily during the first postoperative week and was then stopped. The HUS did not recur, and the plasma creatinine level 24 months post-transplant was $0.9 \mathrm{mg} / \mathrm{dL}(80 \mu \mathrm{mol} / \mathrm{L})$. From that time onwards, the patient developed a progressive fatigue, polypnea and hypoxia without any clinical or laboratory sign of HUS recurrence. No infectious etiology was found. Ultrasound of the heart led to a diagnosis of pulmonary hypertension. The chest X-ray showed diffuse increased vascular prints. The first of the two highresolution computed tomography scans of the lung that were performed led to a suspicion of embolism in one pulmonary artery, but this diagnosis could not be confirmed in subsequent tests. The patient was transferred to the University of Groningen for further diagnostic investigations and was placed on the waiting list for lung transplantation. Due to rapid worsening of hypoxia despite maximal oxygen administration and sildenafil treatment, the patient expired from cardio-respiratory insufficiency. Necropsy analysis of the lungs showed a massive endothelial proliferation in post-capillary venules, strongly indicating a diagnosis of pulmonary veno-occlusive disease (PVOD). Etiological investigation of a suspected pulmonary embolism led to the finding of hyperhomocysteinanemia (plasma concentration: $185 \mu \mathrm{g} / \mathrm{L}$ ). Subsequent DNA sequence analysis of the methylmalonic aciduria cblC with homocystinuria type $C$ gene (MMACH, NM_015506.2) revealed two heterozygous variants: c.276G $>$ T; p.(Glu92Asp)/erroneous splicing and c.442_444delinsA; p.(Val148MetfsX33). The 
c.276G $>\mathrm{T}$ represents the last base of an exon, and the mutation is thus predicted to cause erroneous splicing, as indeed indicated by several splice prediction tools (e.g. http://www.fruitfly.org/seq_tools/splice.html). To the best of our knowledge, both variants are novel and should be considered pathogenic due to their nature of the mutation (frameshift/erroneous splicing). Moreover, the variants were not detected in 210 control alleles. DNA sequence analysis of the parents confirmed compound heterozygosity in the affected child. Several mutations corresponding to different subgroups of $\mathrm{Cbl}$ (cobalamin) disorders have been identified. Depending on the subgroup, there may be a processing defect in either the remethylation of homocysteine to methionine or the conversion of L-methylmalonyl-CoA to succinate, or in both mechanisms, leading to hyperhomocysteinemia, methylmalonylaminoacidemia or the association of both metabolic abnormalities, respectively. It should be noted that the cblC disorder is the most common inborn error of $\mathrm{Cbl}$ metabolism. Several cases of aHUS associated with cobalamin disorders have already been reported, and these were recently reviewed in Pediatric Nephrology [3]. Two different types of cblC MMACHC gene mutations leading to early (neonatal) and late onset aHUS (as in our patient), respectively, have been described (for review, see [3]).

The unusual course of renal function for aHUS associated with $C D 46$ mutations observed in this patient confirms previous observations suggesting that the latter lead to ESRF only in presence of genetic and/or environmental modifiers of disease expression, such as combined mutations [2].

Hyperhomocysteinemia-induced damage to glomerular endothelium has been suggested as the putative mechanism for cblC-associated HUS [3, 4]. Homocysteine alters the antithrombotic properties of the vascular endothelium, possibly through impairing the nitric oxide-mediated inhibition of platelet aggregation [4]. In our patient, the already intrinsically fragile membrane of the endothelial cells resulting from the $C D 46$ mutation was exposed to the toxicity of high plasma concentrations of homocysteine resulting from the MMACHC gene mutations. The lack of HUS recurrence after transplantation confirms the role of the interaction of both mechanisms on the glomerular endothelium since the transplanted kidney most likely did not present any CD46 mutation. Following kidney transplantation, the anti-calcineurin endothelial toxicity of cyclosporine may have been the ultimate triggering factor for endothelial cell proliferation in pulmonary venules.

In conclusion, this case report suggests that the progressive on-going HUS leading to ESRF as well as the fatal PVOD observed in this patient probably resulted from the combination of different pathogenic mechanisms of endothelial dysfunction associated with $C D 46$ and cblC $M M A C H C$ gene mutations. It clearly indicates that, in addition to performing mutation analyses for genes coding for complement factors and antibodies directed against the latter, investigations aimed at identifying $\mathrm{Cbl}$ disorders via homocysteine and methylmalonic acid determination and mutation analyses should be added to the work-up of every aHUS case whatever the age of presentation of the patient since clinical manifestations can be delayed and the latter diagnosis may have important clinical and therapeutic implications.

Open Access This article is distributed under the terms of the Creative Commons Attribution Noncommercial License which permits any noncommercial use, distribution, and reproduction in any medium, provided the original author(s) and source are credited.

\section{References}

1. Davin JC, Buter N, Groothoff JW, van Wijk J, Bouts AH, Strain L, Goodship T (2009) Prophylactic plasma exchange in CD46associated atypical haemolytic uremic syndrome. Pediatr Nephrol 24:1757-1760

2. Richards A, Liszewski MK, Kavanagh D, Fang CJ, Moulton E, Fremeaux-Bacchi V, Remuzzi G, Noris M, Goodship THJ, Atkinson JP (2007) Implications of the initial mutations in membrane cofactor protein (MCP; CD46) leading to atypical hemolytic uremic syndrome. Mol Immunol 44:111-122

3. Sharma AP, Greenberg CR, Prasad AN, Prasad C (2007) Hemolytic uremic syndrome (HUS) secondary to cobalamin C (cblC) disorder. Pediatr Nephrol 22:2097-2103

4. Stamler JS, Osborne JA, Jaraki O, Rabbani LE, MullinsM SD, Loscalzo J (1993) Adverse vascular effects of homocysteine are modulated by endothelium-derived relaxing factor and related oxides of nitrogen. J Clin Invest 91:308-318 